\title{
EARLY COMPLICATIONS OF INGUINAL HERNIA REPAIR
}

\author{
DR. MIRZA ASADULLAH BEG \\ Assistant Professor of Surgery Jinnah Medical \\ College Hospital, Karachi. \\ DR. SYED HUSSEIN MEHDI \\ Associate Professor of Surgery Jinnah Medical \\ College Hospital, Karachi.
}

\author{
DR. SHEERAZ SHAKOOR SIDDIQUI \\ Assistant Professor of Surgery \\ Jinnah Medical College Hospital \\ Karachi.
}

\begin{abstract}
Introduction: Although most complications are of a mild variety, some severe, potentially life-threatening complications have also been reported such as bleeding from the femoral vessels. Period: From January till July 2005. Materials\& Methods All adult patients admitted for inguinal hernia repair on an elective basis were included. Records of hernioplasties done were also incorporated in the database. Exclusion criteria Age under 14 years, emergency surgery and incomplete records. Results: A total of 205 elective inguinal hernioplasties were performed. The complications were encountered classified into three categories; Pei-operative, Anesthesia-related \& Post-operative. Conclusion: We propose that all future research categorize the complications according to the proposed causes so as to better streamline the future guidelines.
\end{abstract}

\section{INTRODUCTION}

Surgical treatment of inguinal hernias has undergone tremendous transformation in the past hundred years. And yet, not even the most modern and sophisticated forms of repair are free of morbidity and recurrence ${ }^{12}$. Although most complications are of a mild variety, some severe, potentially life-threatening complications have also been reported such as bleeding from the femoral vessels1. Also, the spectrum of complications remains unchanged regardless of the method repair chosen.

Fortunately the problem has been addressed well in the local literature. The overall morbidity reported from around the country ranges between 5 and $30 \%{ }^{3 ; 4}$. It is heartening to note that most surgeons indulging in hernia research in this part of the world strongly recommend tension-free mesh hernioplasty as the gold-standard. ${ }^{4,5}$ treatment

The Jinnah Medical College Hospital is a tertiary care facility that caters to a significant proportion of the local population of Korangi. One out of every 'our operations performed at our Center is groin hernid surgery. This study is based on the overall experience with respect to morbidity associated with inguinal hernioplasty in the past two years. 


\section{MATERIALS \& METHOD}

This was a mixed retrospective f,nd prospective study. All adult patients admitted for inguinal hernia repair on an elective basis from January till July 2005 was included. Additionally, records of hernioplasties done from July 2003 till December 2004 were also incorporated in the database.

Exclusion criteria were as follows: presence of dual pathology (e.g. hernia with hydrocele), age under 14 years, emergency surgery and incomplete records. All data was entered on assigned proformas that included per-operative findings, daily postoperative records (in-patient), and follow-up details (out-patient), the latter undertaken at $2 \mathrm{~d}$ and 4 th week post-operatively.

Early complication was defined as that occurring within one month of surgery, as direct result of either the surgical procedure or/and anesthesia.

A prototype of the proforma was created on the SPSS and results were accordingly calculated on the same program.

\section{RESULTS}

A total of 205 elective inguinal hernioplasties were performed in the afore-mentioned study period. Of these, two were excluded for want of insufficient data. Pie charts 1 and 2 summarize the demographic details related to gender and age respectively. Table I summarizes the side of hernia, along with the type of anesthesia used. Standard on-lay mesh repair with a polypropylene sheet $(6 \times 11 \mathrm{cms})$ was affected in all cases. We arbitrarily classified complications into three categories:

\section{Per-operative}

Anesthesia-related; \& Post-operative.

Per-operative: Two patients sustained transaction of the vas, while one developed diathermy burns.

Anesthesia-related: As shown in table I, a significant majority was operated upon under spinal anesthesia. In this group $(\mathrm{n}=140), 10$ patients developed urinary retention (7.1\%). An almost equal number suffered

through spinal headaches for an average of 4.5 days post-operatively $(n=12,8.5 \%)$

In the general anesthesia group $(n=50), 5$ patients developed post-operative urinary retention and another 5 sustained pulmonary insufficiency, mainly atelectasis (10\% each). No morbidity was observed in the local-infiltration group $(n=13)$.

Table III summarizes the overall complication rate of anesthesia.

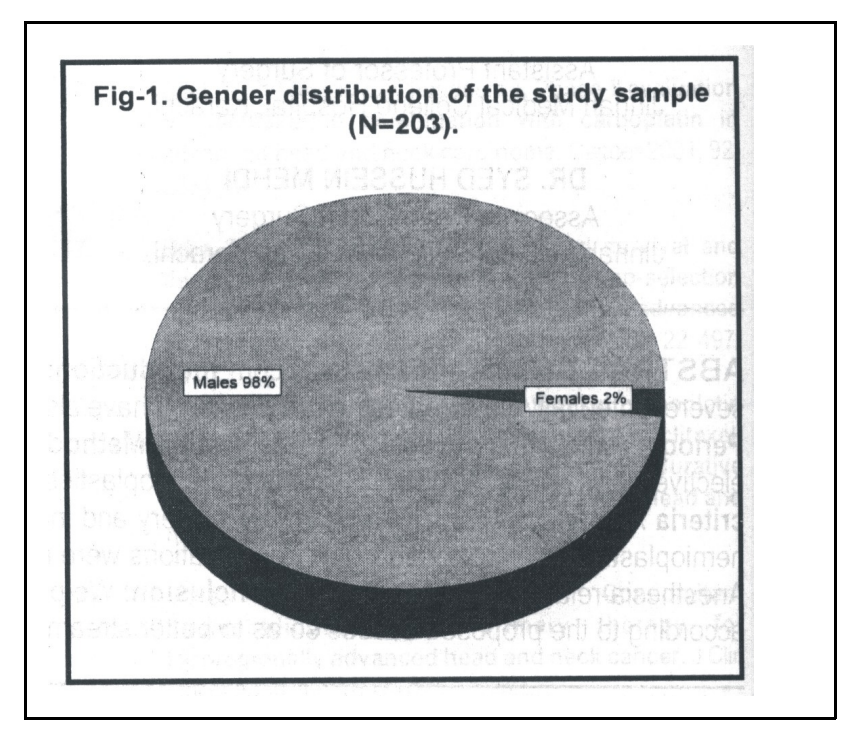

\begin{tabular}{|l|c|c|c|c|}
\hline \multicolumn{5}{|c|}{ Table-1. Type of anesthesia used and side of defect in the } \\
sample (N=203). \\
\hline & $\begin{array}{c}\text { Spinal } \\
\text { Anae. }\end{array}$ & $\begin{array}{c}\text { General } \\
\text { Anae. }\end{array}$ & $\begin{array}{c}\text { Local } \\
\text { Anae. }\end{array}$ & Total \\
\hline Left side & 74 & 21 & 5 & 100 \\
\hline Right side & 65 & 20 & 8 & 93 \\
\hline Bilateral & 1 & 9 & - & 10 \\
\hline Total & 140 & 50 & 13 & 203 \\
\hline
\end{tabular}


Post-operative: Wound seromas complicated 24.6\% cases $(n=50)$. It came out as the commonest individual morbidity in our series. Conservative measures were employed in all cases, avoiding even needle drainage owing to the prosthesis.

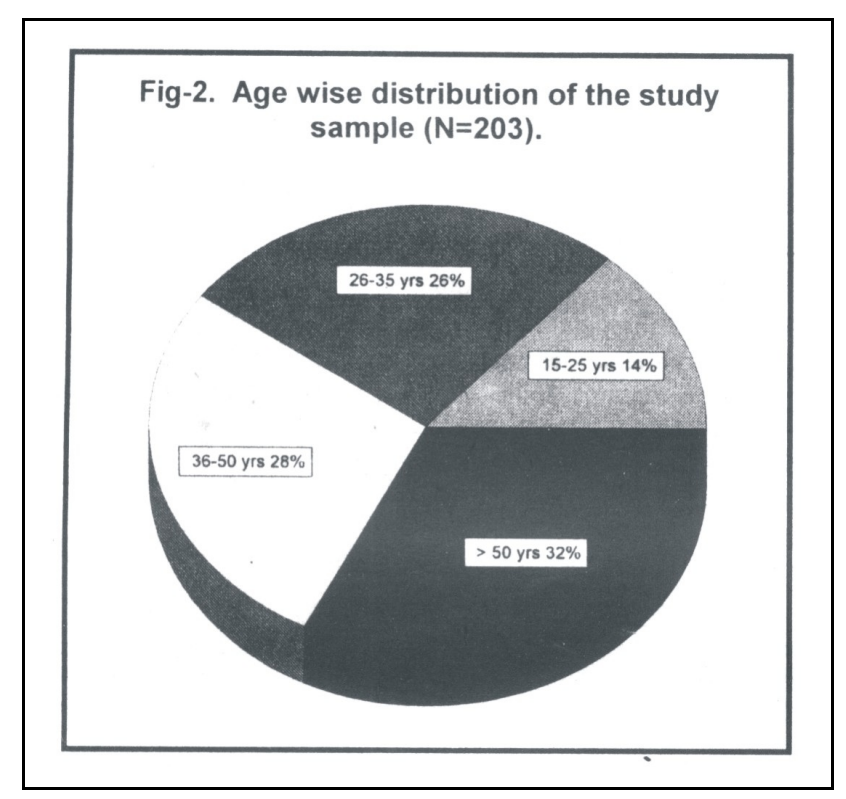

Ischemic orchitis (diagnosed clinically by the presence of swollen, painful testes) developed in 12 patients $(5.9 \%)$. The mean duration of appearance of symptoms was 7.5 days after surgery. Again, only conservative means settled the problem.

Neurogenic symptoms (burning pain, hypo and hyper-anesthesia) were encountered in 4 patients ( 1 $.9 \%)$. Two patients with unrelenting pain were given the option of re-exploration, which they declined.

Luckily, only two patients developed wound infection, of which one required removal of prosthesis and repeated lavage. Delayed primary repair without a mesh was undertaken in this case, an elderly diabetic male. The other gentleman settled on conservative therapy.

Overall Morbidity: The overall morbidity was
$49.7 \%(n=101)$

\section{DISCUSSION}

In our series, we have arbitrarily categorized complications into the three types vis per-operative, anesthesia-related and post-operative. We strongly feel that such categorization, although unprecedented in the local and international literature, yields more fruitful results in terms of steps to limit morbidity. Further, the morbidity factor must take into account both the etiology and chronology of an event. Otherwise, simple figures may not contribute much to the formulating future guidelines towards safe surgery.

In our study, localized fluid collections (so-called seromas) topped the list of morbidity. We believe over-enthusiastic use of the Bovie to be the responsible cause. Seromas after groin hernia surgery have been universally reported, albeit at much lower frequencies ${ }^{23}$. Aziz et $\mathrm{al}^{5}$ in a series of 100 cases encountered this problem in a decent $9 \%$ only. Interestingly though, most of the local literature reports these collections along with hematomas and scrotal swellings ${ }^{35^{\prime} 6}$, we only came across localized serous collections and no cases of either of these problems. The discrepancy reflects the often nebulous and overlapping criteria employed to designate a collection as a seroma, a hematoma or simple postoperative edema. More standard definitions need to be formulated to differentiate these terms.

Our rate of wound-sepsis, although limited, was annoying considering the fact that we routinely use prosthetic mesh for the repair. Internationally, the infection rate stands at a well-controlled ${ }^{0.6 \% 7}$.

Although the overall morbidity was a staggering $49.7 \%$, the rest of the spectrum of complications was accounted for only by a limited number of individual figures as presented in the results section. Also, we did not encounter most of the 
complications that have otherwise been so commonly reported in the international and national literature; namely, urinary retention, vas transection or other anesthesia-related morbidity.

\section{CONCLUSION}

Morbidity of inguinal hernia surgery presents a wide spectrum of complications. Not all of these may be related to the surgical procedure. We propose that all future research categorize the complications according to the proposed causes so as to better streamline the future guidelines. This is especially relevant in the backdrop of everincreasing use of the prosthetic mesh.

\section{REFERENCES}

1. BendavidR. Complications of groin hernia surgery. U ClinNAm. 1998; 78:1089-1103.

2. Barbir J, Carretier M, Richer JP. Cooper ligament repair: an update. World J Surg. 1989; 13:499-505.
3. Aurangzeb M. Tension-free hernioplasty: A review of 96 cases. J Post-grad Med Inst. 2004; 18:46-51.

4. Aslam M, Arain GM, Khan SA. Inguinal hernia day case surgery: Study of 100 cases. Biomedica. 2002; 18:70-3.

5. Aziz M, Ahamad N, Anwar F, Faizullah. Comparative study of post-operative complications of Lichtenstein tension-free repair and pure-tissue repairs like modified Bassini or Shouldice at Nioshtar Hospital, Multan. Ann King Edward Med Coll. 2004:10: 39-42.

6. Khan Z, Gardezi JR. Lichtenstein tension-free hernioplasty: An Audit. Pakistan J Surg. 2004; 20:16-19.

7. Amid PK, Schulman AG, Lichtenstein IL. The Lichtenstein open tension-free hernioplasty. In: Inguinal Hernia: Advances or controversies. Oxford, England, Radcliff Med Press. 1994; pp 107-116. 